doch erhöht. Deshalb sei es nicht nachvollziehbar, dass diese schlechter bezahlt würden. „Diese Klage werden wir wieder zurückziehen“, kündigte KBV-Chef Dr. Andreas Gassen an. Er zeigte sich zufrieden, dass „unser Engagement für die psychotherapeutische Versorgung der Patienten erfolgreich gewesen“" sei. „Ich freue mich, dass die Kassenseite ihre
Position noch einmal überdacht hat und nachgebessert werden konnte.“

Im ersten Vergütungsbeschluss des Erweiterten Bewertungsausschusses vom 29. März 2017, der gegen die Stimmen der KBV gefallen war, waren für die Sprechstunde (EBM-Nr. 35151) sowie für die Akutbehandlung (EBM-Nr. 35152) bei einer Dauer von mindestens $25 \mathrm{Mi}$ - nuten $42,75 €$, bei 50 Minuten $85,50 €$ vorgesehen. Rückwirkend zum 1. April 2017 erhalten die Vertragsärzte und -psychotherapeuten nun für eine Sitzung von mindestens 25 Minuten 44,33€ und bei einer Sitzungsdauer von 50 Minuten $88,56 €$. Das entspricht einer Therapiestunde im Rahmen der Richtlinien-Therapie.

Christoph Winnat

\title{
Rettungsdienst: Gericht bestätigt Selbstständigkeit der Honorarärzte
}

\section{Eine Änderung im Sozialgesetzbuch IV stellt jetzt die Rettungskräfte auf Honorarbasis besser.}

$\stackrel{0}{0}$ berraschend hat kürzlich das Landessozialgericht NRW die nebenberufliche Notarzttätigkeit auf Honorarbasis als selbstständig eingestuft (Az.: L 8 R 162/15). Das stelle „eine grundsätzliche Abkehr von den Entscheidungen aus Niedersachsen und MecklenburgVorpommern dar", kommentiert der Kölner Anwalt Dr. Stephan Porten das noch unveröffentlichte Urteil. Dort hatten Gerichte Rettungskräfte auf Honorarbasis als abhängig Beschäftigte klas- sifiziert, für die Sozialabgaben zu zahlen sind. Das führte schließlich zu einer Änderung im Sozialgesetzbuch IV, wonach bei einer Haupttätigkeit über mindestens 15 Wochenstunden oder bei Niederlassung in eigener Praxis Honorarnotärzte von der Abgabepflicht befreit sind.

Dem Essener Urteil komme mehr als nur rechtsgeschichtliche Bedeutung zu, so Porten: Derzeit würden viele Betriebsprüfungen bei den Diensten laufen, denen hohe Nachforderungen der Sozi- alversicherung folgen könnten. Dagegen sei das Urteil eine gute Argumentationshilfe. Und weil sich am Status der von mehreren Gerichten bestätigten abhängigen Beschäftigung durch die sozialrechtliche Ausnahme ja nichts ändere, seien Rettungsdienstträger in der Praxis mit weiteren Problemen konfrontiert, wie etwa den Ruhezeitvorschriften oder der lohnsteuerlichen Veranlagung. Auch diesbezüglich könne der Essener Richterspruch Signalwirkung entfalten.

Die neu geschaffene Ausnahme greift nur für die Zukunft, bei Bedarf sind neue Verträge zu schließen. Denn eine Übergangsregelung ( $\$ 118$ SGB IV) besagt, dass die Abgabenfreiheit „nicht für Einnahmen aus einer vor dem 11. April 2017 vereinbarten Tätigkeit als Notarzt im Rettungsdienst" gilt. Christoph Winnat

\section{Zuerst der genaue Blick in die Bücher, dann die Praxisübernahme}

\section{Was Ärzte bei der Übernahme einer Praxis zur Existenzgründung beachten sollten, spiegelten Niederlassungsexperten in der "KarriereSprechstunde".}

\footnotetext{
$\ddot{A}$ rzte, die zur Existenzgründung eine Praxis übernehmen wollen, müssen nicht darauf warten, bis sie eine finden, die mit denselben Schwerpunkten geführt wird, wie sie sich das selbst vorstellen. Darauf verwiesen Niederlassungsexperten in der „KarriereSprechstunde“ im Mai 2017 in Stuttgart. „Nach ein paar Jahren machen Sie sich die Praxis ohnehin nach Ihren eigenen Schwerpunkten“, betonte Medizinrechtler und Praxisberater Dr. Lars Lindenau, ETL ADVISION. Vor der Praxisübernahme ist allerdings ein genauer Blick in die
}

Finanzunterlagen notwendig - vor allem in die Einnahmen-Überschussrechnung sowie in die betriebswirtschaftlichen Auswertungen des laufenden Jahres und der vergangenen drei Jahre. So kann die wirtschaftliche Tragfähigkeit der Übernahme fundiert beurteilt werden, bestätigte Klaus-Jürgen Bayer, Filialleiter der apoBank Stuttgart. Wichtig seien auch die Honorarbescheide der $\mathrm{KV}$, aus denen die Einnahmestruktur der Praxis hervorgehe, wie Heike Müller-Henkel, KV Baden-Württemberg, betonte.
Anfangs sei es von Vorteil, wenn der Übernehmer die Weiterbildungen habe, die nötig seien, um spezielle Leistungsschwerpunkte des Vorgängers zu bedienen. „Letztlich müssen auch alle Verträge auf den Tisch“, sagte Lindenau auf der Veranstaltung von der apoBank, ETL ADVISION und Springer Medizin. Nur so würden die tatsächlichen Kostenstrukturen sichtbar. „War die Ehefrau angestellt? Welche Qualifikationen haben die Fachangestellten? Ist die Praxis wie bisher in den Räumen fortführbar, etwa wenn die Immobilie dem Vorgänger gehört? Welche Perspektiven bietet der Mietvertrag?" Das seien wichtige Fragen, deren Antworten für die Einschätzung der Zukunft der Praxis essenziell seien. Lindenau: „Läuft die Praxis dann einmal, können Sie Ihre eigenen Schwerpunkte setzen."

Hauke Gerlof 